\title{
High-dose dobutamine stress magnetic resonance perfusion imaging at 3.0 Tesla
}

\author{
Rolf Gebker ${ }^{1 *}$, Alexander Berger ${ }^{1}$, Christopher Schneeweis ${ }^{1}$, Thomas Hucko ${ }^{1}$, Bernhard Schnackenburg ${ }^{2}$, \\ Christoph Klein ${ }^{1}$, Sebastian Kelle ${ }^{1}$, Eckart Fleck ${ }^{1}$ \\ From 16th Annual SCMR Scientific Sessions \\ San Francisco, CA, USA. 31 January - 3 February 2013
}

\section{Background}

Dobutamine stress magnetic resonance (DSMR-wall motion) has been established as a valuable tool for the detection of inducible wall motion abnormalities. Additional perfusion imaging during DSMR (DSMR-perfusion) was shown to improve sensitivity for the detection of myocardial ischemia. Current experience is based on 1.5T only, mainly due to insufficient image quality of steady state free precession (SSFP) cine imaging at higher field strengths. Recently, image quality and diagnostic accuracy of SSFP sequences was significantly improved by dual-source parallel radiofrequency (RF) transmission for 3.0T. We examined whether the addition of myocardial perfusion imaging during DSMR at 3.0T is feasible and whether it provides incremental benefit for the evaluation of CAD.

\section{Methods}

DSMR-wall motion and -perfusion were combined in 48 consecutive patients on 3.0T clinical systems (Philips Achieva and Ingenia, both equipped with a dual-source RF transmission system) applying a standard high dose dobutamine-atropine protocol. Perfusion images were acquired

in three short axis views during maximum stress. Wall motion and perfusion images were interpreted sequentially by two blinded readers with invasive coronary angiography serving as the reference standard.

\section{Results}

Assessment of wall motion and perfusion at rest and during stress were feasible in $98 \%$ of patients. One patient (2\%) developed atrial fibrillation during dobutamine infusion and the examination had to be terminated. Two patients (4\%) developed wall motion abnormalities before reaching target heart rate and DSMR-perfusion was performed at this time. Mean dobutamine dose was $32 \pm 8$ $\mu \mathrm{g} / \mathrm{kg} / \mathrm{min}$, atropine was given in $28(58 \%)$ patients. Interobserver agreement was very good for DSMR-wall motion $(\kappa 0.88)$ and -perfusion $(\kappa 0.83)$. The prevalence of CAD ( $>70 \%$ stenosis) was $64 \%$ and involved 45 coronary territories. On a patient basis, diagnostic accuracy of DSMR-wall motion and DSMR-perfusion were similar (Table). DSMR-wall motion correctly identified 28 (62\%) territories supplied by a stenotic coronary artery, whereas DSMR-perfusion correctly identified significantly more

Table 1 Diagnostic accuracy of DSMR-wall motion and DSMR-perfusion

\begin{tabular}{|c|c|c|c|c|c|c|c|c|c|}
\hline & \multicolumn{3}{|c|}{ Sensitivity } & \multicolumn{3}{|c|}{ Specificity } & \multicolumn{3}{|c|}{ Accuracy } \\
\hline $\begin{array}{c}\text { Coronary } \\
\text { stenosis } \geq 50 \%\end{array}$ & DSMR-wall motion & DSMR-perfusion & $P$ & DSMR-wall motion & DSMR-perfusion & $P$ & DSMR-wall motion & DSMR-perfusion & $P$ \\
\hline All patients & $27 / 36(75)$ & $30 / 36(83)$ & 0.25 & 10/11 (91) & 10/11 (91) & 1 & $37 / 47(79)$ & $40 / 47(85)$ & 0.38 \\
\hline $\begin{array}{c}\text { Coronary } \\
\text { stenosis } \geq 70 \%\end{array}$ & & & & & & & & & \\
\hline All patients & 26/30 (87) & 28/30 (93) & 0.5 & 15/17 (88) & 14/17 (82) & 1 & 41/47 (87) & 42/47 (89) & 0.38 \\
\hline
\end{tabular}

values are $\mathrm{n}(\%), \mathrm{P}$ indicates the significance level for differences between DSMR-wall motion and DSMR-perfusion

${ }^{1}$ German Heart Institute Berlin, Berlin, Germany

Full list of author information is available at the end of the article

(c) 2013 Gebker et al; licensee BioMed Central Ltd. This is an Open Access article distributed under the terms of the Creative Commons 


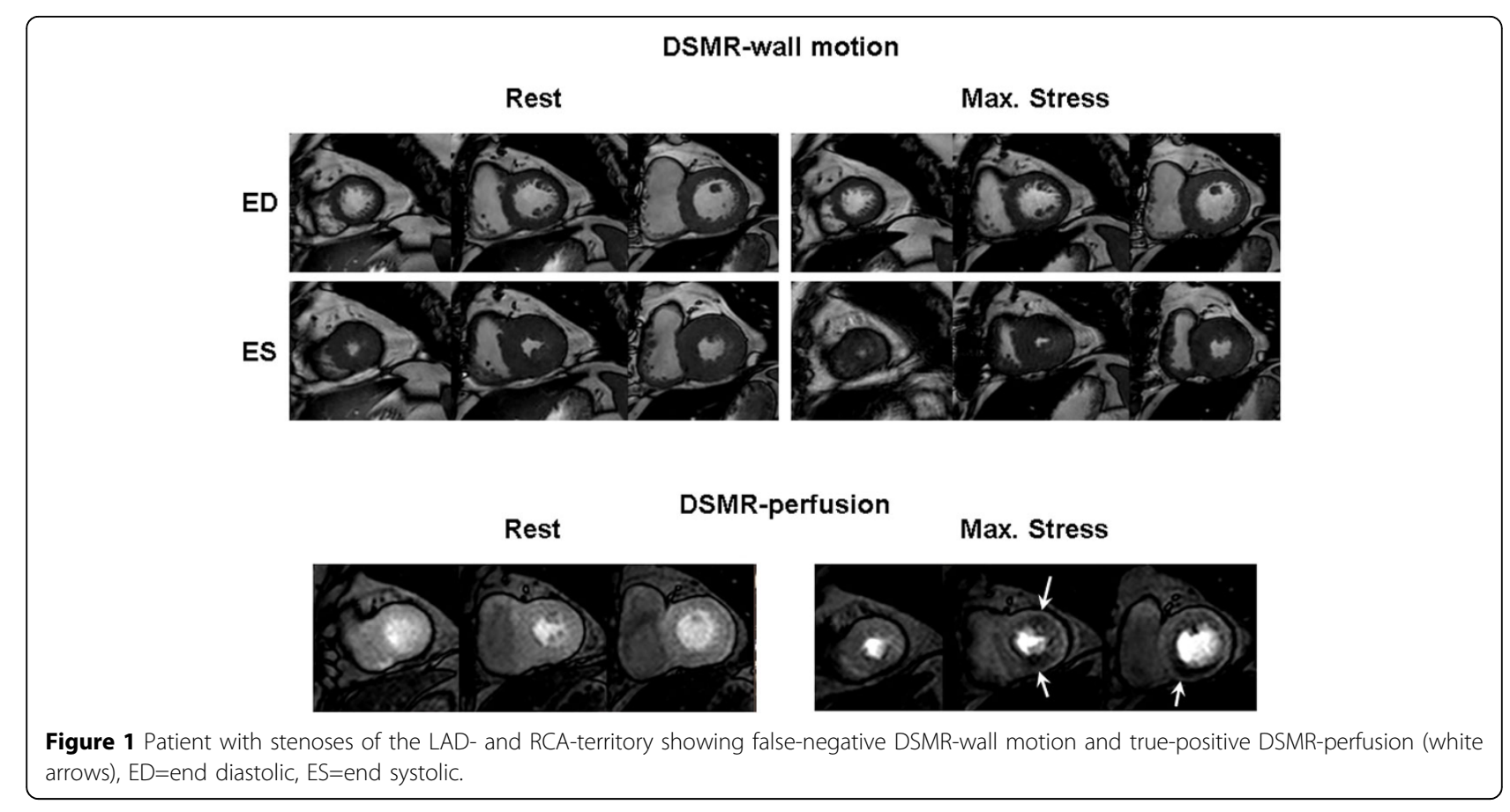

ischemic territories $(\mathrm{n}=38,84 \%, \mathrm{P}<0.002)$. The ability to recognize a patient as having multivessel disease by more than one territory demonstrating an ischemic reaction was improved significantly by use of DSMR-perfusion (91\% vs. $36 \%, p=0.03$, Figure).

\section{Conclusions}

DSMR-perfusion is feasible with a high procedural success rate at 3.0T applying dual-source RF transmission and has additional diagnostic value compared to DSMR-wall motion regarding the extent of stress inducible ischemia.

\section{Funding}

none.

\section{Author details}

${ }^{1}$ German Heart Institute Berlin, Berlin, Germany. ${ }^{2}$ Philips Health Care,

Hamburg, Germany.

Published: 30 January 2013

doi:10.1186/1532-429X-15-S1-P211

Cite this article as: Gebker et al:: High-dose dobutamine stress magnetic resonance perfusion imaging at 3.0 Tesla. Journal of Cardiovascular Magnetic Resonance 2013 15(Suppl 1):P211.

Submit your next manuscript to BioMed Central and take full advantage of:

- Convenient online submission

- Thorough peer review

- No space constraints or color figure charges

- Immediate publication on acceptance

- Inclusion in PubMed, CAS, Scopus and Google Scholar

- Research which is freely available for redistribution 